\title{
Reflexões sobre a identificação de níveis de affordances em interfaces bancárias digitais
}

\author{
Reflections on the identification of affordances levels in digital banking interfaces
}

Dominique Leite Adam, André Schlemmer, Stephania Padovani, Maria Lucia Leite Ribeiro Okimoto

processamento de informação, affordances, design de interação, design centrado do usuário

As interfaces digitais podem mediar as relações entre humano e computador em diferentes áreas da sociedade. No design, a mediação pode ocorrer por meio de interações, que são projetadas para serem percebidas, compreendidas, e auxiliarem na tomada de decisão dos usuários. À vista disso, o presente artigo tem como objetivo apresentar uma reflexão a respeito dos níveis de affordances em interfaces bancárias sob a ótica do Design da Informação. Os procedimentos metodológicos são norteados por um levantamento bibliográfico acerca do processamento de informações e níveis de affordances, seguido de uma análise de identificação das affordances a partir de relatos de experiências dos usuários e exemplificação de interface. Os resultados sugerem que, em geral, as affordances são percebidas pelos usuários, porém demandam um esforço cognitivo adicional para compreendê-las. A partir dessas reflexões, conclui-se que o designer precisa considerar a familiaridade e experiência dos usuários para aplicar os níveis de affordances em seus projetos.

information processing, affordances, interaction design, user-centered design

Digital interfaces can mediate the human-computer relationship in different areas of society. In design, mediation can occur through interactions, which are designed to be perceived, understood, and assist users in making decisions. Given this, this article aims to present a reflection on the levels of affordances in banking interfaces from the Information Design's perspective. The methodological procedures are guided by a bibliographic review about the processing of information and levels of affordances, followed by an analysis of identification of affordances from users' experiences reports and an example of an interface. The results suggest that, in general, affordances are perceived by users, but require additional cognitive effort. From these reflections, it is concluded that the designer needs to consider the familiarity and experience of the users to apply the affordances levels in their projects.

\section{Introdução}

A relação do humano com o ambiente é mediada por recursos físicos e digitais que influenciam diretamente o modo em como percebemos o contexto. A percepção faz com que o usuário reconheça, observe e discrimine os estímulos do ambiente a fim de associar as informações no contexto e adquirir experiências com base em aspectos comportamentais, motivacionais e culturais (Shedroff, 1999; Norman, 2013).

Anais do $10^{\circ} \mathrm{CIDI}$ e $10^{\circ} \mathrm{CONGIC}$

Kelli C.A.S. Smythe, Rafael de Castro Andrade (orgs.)

Sociedade Brasileira de Design da Informação - SBDI

Curitiba | Brasil | 2021
Proceedings of the $10^{\text {th }} \mathrm{CIDI}$ and $10^{\text {th }}$ CONGIC

Kelli C.A.S. Smythe, Rafael de Castro Andrade (orgs.)

Sociedade Brasileira de Design da Informação - SBDI Curitiba | Brazil | 2021 
Durante o processo de desenvolvimento, os designers exploram problemas práticos em busca de soluções que sejam compreensíveis aos usuários, porém ao elaborar o modelo conceitual do projeto existe uma relação que depende da capacidade do usuário em absorver tais informações (modelo mental do usuário). Dessa forma, é importante o designer considerar o processamento de informação (vide figura 1), que envolve aspectos viscerais, comportamentais e reflectivos, que irão influenciar nas escolhas de projeto, e consequentemente nas tomadas de decisão dos usuários (Norman, 2013).

Figura 1: Processamento da informação | Fonte: Os autores, com base em Norman (2013) e Shedroff (1999)

\section{Processamento da Informação}

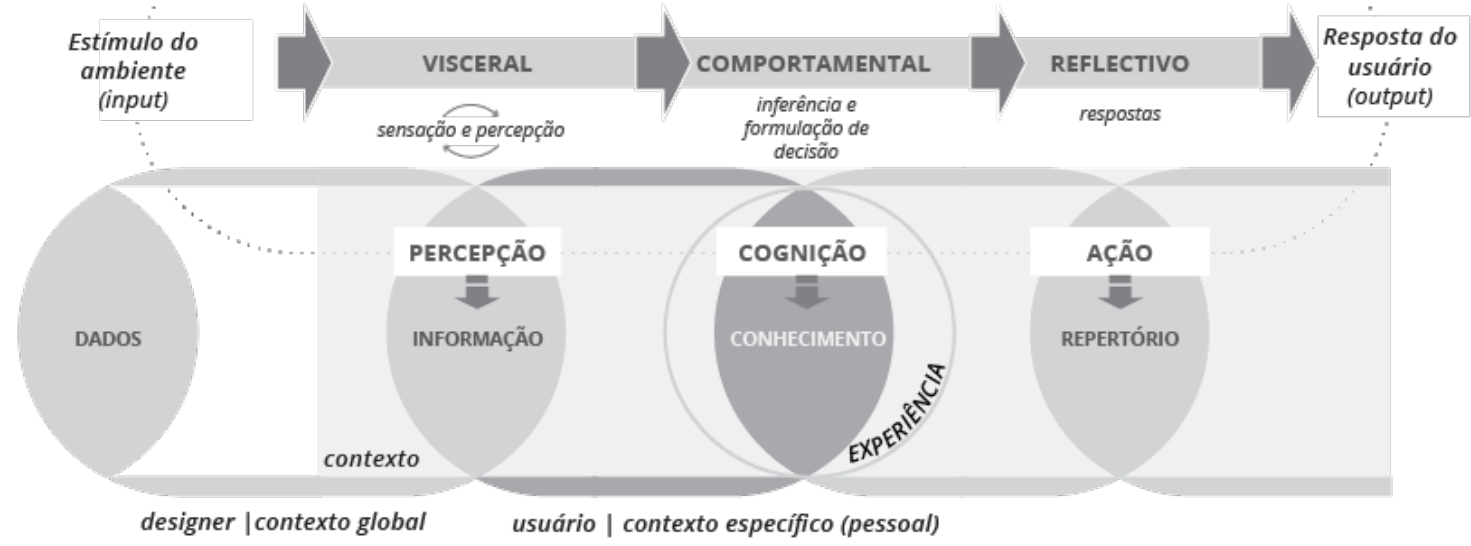

Cabe ressaltar que durante a revisão de literatura foi possível identificar a apresentação dos níveis de processamento em Norman (2004), porém em sua obra mais recente (Norman, 2013), o autor retoma os níveis de processamento em uma abordagem pela qual está estritamente relacionada com os estágios de ação e um dos fundamentos é a affordance. À vista disso, este estudo foi conduzido citando a obra mais recente do autor.

Considerando uma solução compreensível de um problema prático, os designers podem relacionar o conceito de affordance nos projetos de interfaces (o meio para que a interação entre usuário e o artefato aconteçam), por intermédio do desenvolvimento de atributos, propriedades que podem ser físicas ou funcionais. Porém, as affordances não estão focadas nas propriedades em si, mas na relação entre o usuário e o artefato, nas interações essenciais para tornar possíveis e óbvias as ações (Gaver, 1991; Norman, 2013).

Com base em Gaver (1991) e Norman (2013) este estudo adota a definição de affordance ${ }^{1}$ como sendo uma característica física ou digital de determinado artefato que, quando percebida por um usuário, é apreendida, interpretada e gera a consciência de uma resposta (ação) diante

\footnotetext{
${ }^{1}$ Termo originário da área da Psicologia Ecológica, que condiz com a capacidade de ação de um animal e as possibilidades de ações do ambiente (GIBSON, 1986).
} 
o contexto de uso. A facilidade de percepção e interpretação da affordance é dependente do repertório cultural e experiência prévia.

Um exemplo de projeto de design que precisa atender uma diversidade de usuários (aspectos culturais e de experiência) condiz com as interfaces bancárias digitais. Segundo o Relatório de Cidadania Financeira feito pelo Banco Central (2018) as transações nos canais digitais estão em ascensão. As operações bancárias por meio de canais não presenciais correspondem a 66\% das transações. Entre 2015 e 2017 as transações por smartphones cresceram $19 \%$ e representam junto com os PDAs $35 \%$ da quantidade total de transações (remotas e presenciais). Sendo assim, este estudo propõe uma reflexão sobre a influência dos níveis de affordance no reconhecimento das possibilidades de ação em interfaces bancárias digitais.

Diante deste contexto, objetiva-se identificar e classificar os níveis de affordance nas interfaces bancárias digitais. Para tanto, foi realizada uma triangulação entre a teoria de design da informação, experiências vivenciadas por usuários dessas interfaces e exemplificação de uma interface bancária de aplicativo mobile.

\section{Interfaces e Affordances}

No Design, um dos campos que podem ser realizados estudos que envolvam a interface é na interação humano-computador (IHC). Segundo Dix et al. (2004) a IHC está fundamentada em diversas disciplinas, mas as áreas que têm como relevância projeto, implementação e avaliação de sistemas interativos consideram a IHC como uma das preocupações centrais.

Diante deste cenário, uma possibilidade de estudos em Design que envolve a IHC acontece através da interface com o usuário, que pode ser entendida segundo a NBR ISO 9241-210 ABNT (2011, p. 04) como "todos os componentes de um sistema interativo (software ou hardware) que fornecem informações e controles para o usuário realizar tarefas específicas com o sistema interativo".

As interfaces podem ser classificadas conforme o tipo de função, o estilo de interação, o dispositivo de entrada/saída ou a plataforma que será projetada (Rogers, Sharp \& Preece, 2019). Neste estudo, o objeto analisado são as interfaces bancárias, especificamente, o tipo web (site) e mobile (aplicativo), ambas interfaces apresentam recursos e componentes de interação para os usuários interagirem e realizarem suas demandas condizentes com os serviços bancários. De acordo com Galitz (2007) e Sharp, Rogers e Preece (2019), alguns exemplos de recursos e componentes da interface do usuário são: elementos verbais, pictóricos e esquemáticos representados por áreas clicáveis, expansíveis e retráteis, menus, ícones, caixas de diálogo, entre outros que podem ser acionados por meio de diferentes ações, como pinçar, rolar, arrastar, clicar, duplo clique, pressão, toque, entre outros.

A affordance é um dos mecanismos que possibilita a percepção e compreensão dos usuários em relação às tomadas de decisão durante a interação com a interface bancária. Nesse sentido, Gaver (1991) propõe uma estrutura para classificar as affordances, com o 
intuito de entender que as percepções tanto da informação quanto da affordance podem interferir na interpretação do usuário. A figura 2 representa a estrutura.

Figura 2: Classificação da percepção da informação e affordance | Fonte: Os autores, com base em Gaver (1991)

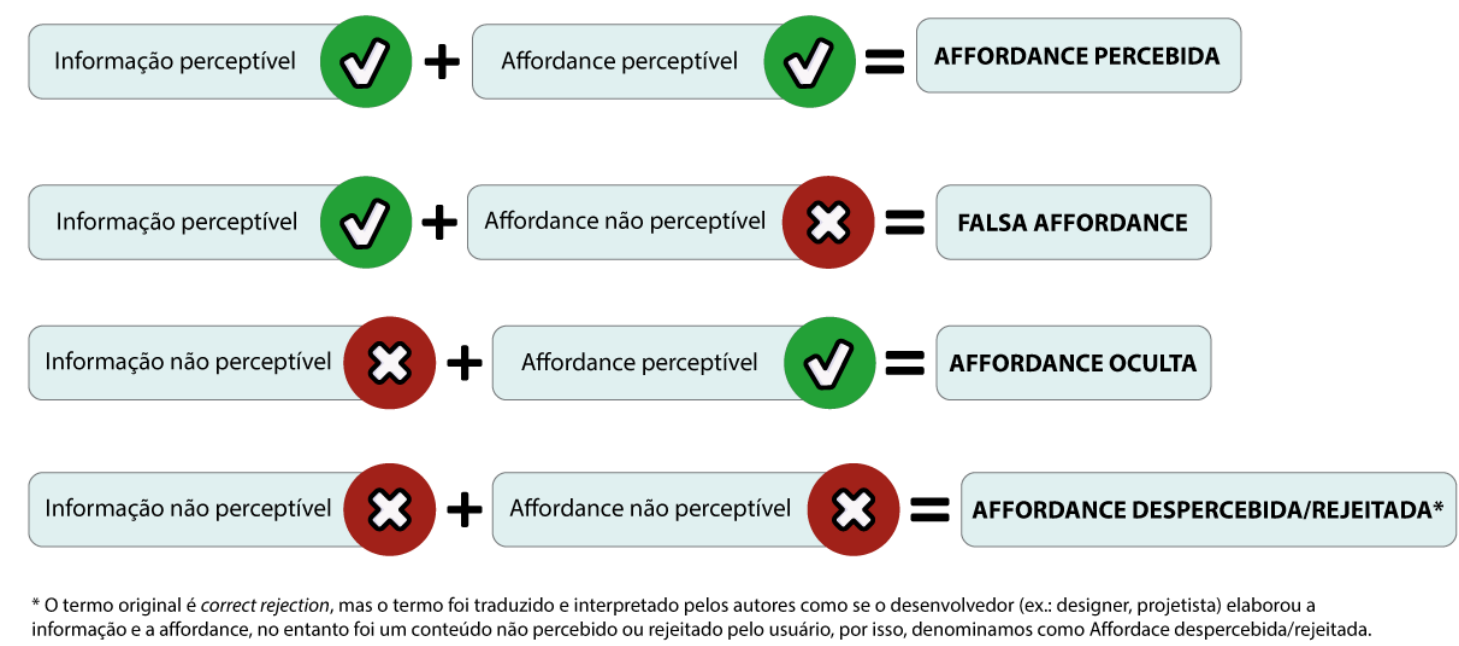

De acordo com a estrutura proposta por Gaver (1991) quando o designer desenvolve uma affordance percebida as informações oferecidas aos usuários, na prática, contribuirão para o percepção e reconhecimento da ação. No entanto, se não tiver a percepção da informação e da affordance, o usuário simplesmente não percebe e nem compreende uma determinada ação, o que pode complicar tanto a facilidade de uso quanto a utilidade da interface (affordance despercebida/rejeitada).

Também existe a possibilidade da interface conter uma informação perceptível, porém com uma affordance inexistente, diante disso, é classificada como falsa affordance (ex.: informar que existe um link e ao clicar no link nada acontece). Outra forma de classificar o nível de affordance é quando a informação não é percebida, apesar da affordance existir. Neste caso, denomina-se affordance oculta (ex.: falta de estímulos gráfico-visuais para indicar área clicável). Em ambas situações podem afetar a compreensão do usuário durante a interação.

McGrenere \& Ho (2000) explicam que o designer precisa compreender que existem dois aspectos correlacionados com a affordance: (1) projetar as affordances e (2) projetar as informações que explicitam as affordances. Por isso, durante a concepção da interface, o designer pode destinar atividades pertinentes no planejamento das affordances, que não basta apenas ter funções corretas (foco na usabilidade), mas ter funções que explicitam as possibilidades de uso compatíveis com o usuário (utilidade percebida).

Diante dessas considerações, o designer precisa de uma orientação para compreender os níveis de affordance que auxiliarão durante a concepção da interface. Através de uma revisão de literatura foi possível identificar três possíveis níveis de affordance em interfaces. O quadro 1 representa os níveis encontrados. 
Quadro 1: Níveis de affordance. | Fonte: Os autores, com base em St. Amant (1999), Kannengiesser e Gero (2012), Hartson (2003)

\section{Níveis de affordance}

\section{St. Amant (1999)}

Simples: a affordance construída pelo designer foi percebida pelo usuário, portanto, a affordance indicou a possibilidade de ação.

\section{Pré-condição de execução:} determina a aplicabilidade da affordance, ou seja, o usuário percebeu, identificou e pode executar a ação.

Execução de efeito: o efeito seria a resposta almejada pelo designer diante da affordance fornecida ao usuário. Portanto, as escolhas do designer influenciam na percepção do usuário e a percepção do usuário influenciam nas escolhas do designer. A relação entre designer $x$ usuário impacta na percepção da affordance.

\section{Avaliação da pré-condição:} propriedades, características (físicas ou digitais) que facilitam o reconhecimento da affordance.

\section{Avaliação de efeito: a} avaliação depende das escolhas do designer e da percepção do usuário para reconhecimento $\mathrm{e}$ tomar decisões perante a affordance, questões como contexto, comandos e ações possíveis de serem realizadas são essenciais para esta avaliação.

\section{Kannengiesser e Gero (2012) Hartson (2003) \\ Reflexivo: perceber e identificar Cognitiva: são recursos de} os estímulos do artefato para auxiliar nas tomadas de decisão do usuário. A affordance neste nível seria em um sentido percebido, sensorial.

Reativo: selecionar, testar, avaliar e reagir perante as possibilidades de ação do artefato. A affordance neste nível seria em um sentido de seleção, ação e atuação.

Reflectivo: interpretar e refletir sobre as diferentes possibilidades de ações da affordance. A affordance neste nível seria no sentido da junção entre a percepção e a cognição que levam a explorar e reformular a nossa ação e atuação com o artefato.

\section{design que permitem o}

reconhecimento da existência da affordance, ou seja, algo existe e serve para alguma coisa.

Física: são recursos de design que auxiliam as tomadas de decisões para realizar uma ação física na interface.

Sensorial: são recursos de design que tornam a affordance perceptível para o usuário, por exemplo, recursos visuais, sonoros, táteis-vibracionais, etc.

Funcional: são recursos de design que tem como característica as funcionalidades da affordance diante do contexto de uso, por exemplo, ícones, botões, cores, feedback sonoro, caixa de diálogo, entre outros.

\section{Método}

Este estudo teórico-prático, de natureza aplicada, compreende um levantamento bibliográfico e análise dos níveis de affordance em interfaces bancárias. Para tanto, foi aplicado um questionário online com usuários deste tipo de interface para coletar possíveis dificuldades de interação. O procedimento de análise dos níveis de affordance ocorreu através da triangulação dos relatos e da exemplificação de interface bancária de um aplicativo mobile.

O estudo foi dividido em três etapas, conforme Figura 3. 
Figura 3: Procedimento metodológico | Fonte: os autores

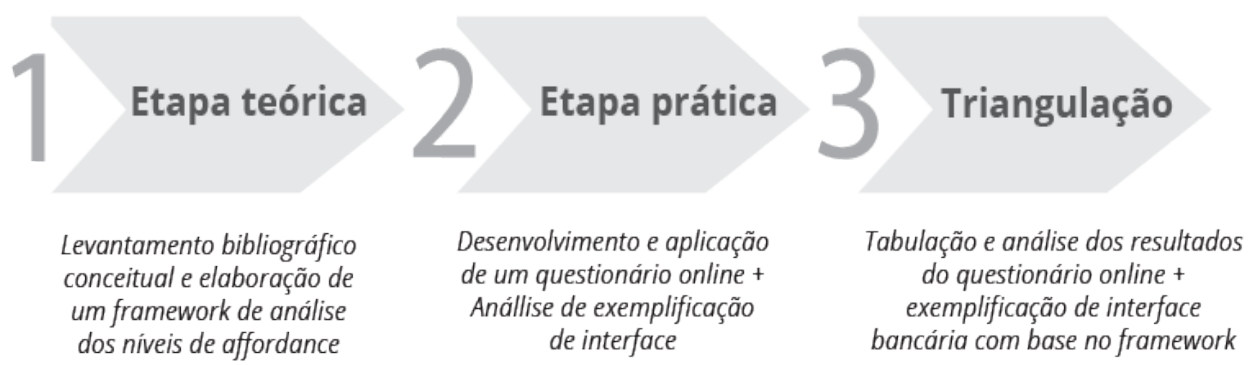

(1) Etapa teórica: Através de uma revisão de literatura foi possível identificar os níveis de affordance e elaborar um framework que pode ser utilizado para mapear esses níveis em interfaces gráfico-informacionais (Figura 4).

Figura 4: Framework de análise dos níveis de affordance | Fonte: Os autores, com base em Hartson (2003), Kannengiesser e Gero (2012) e St. Amant (1999)

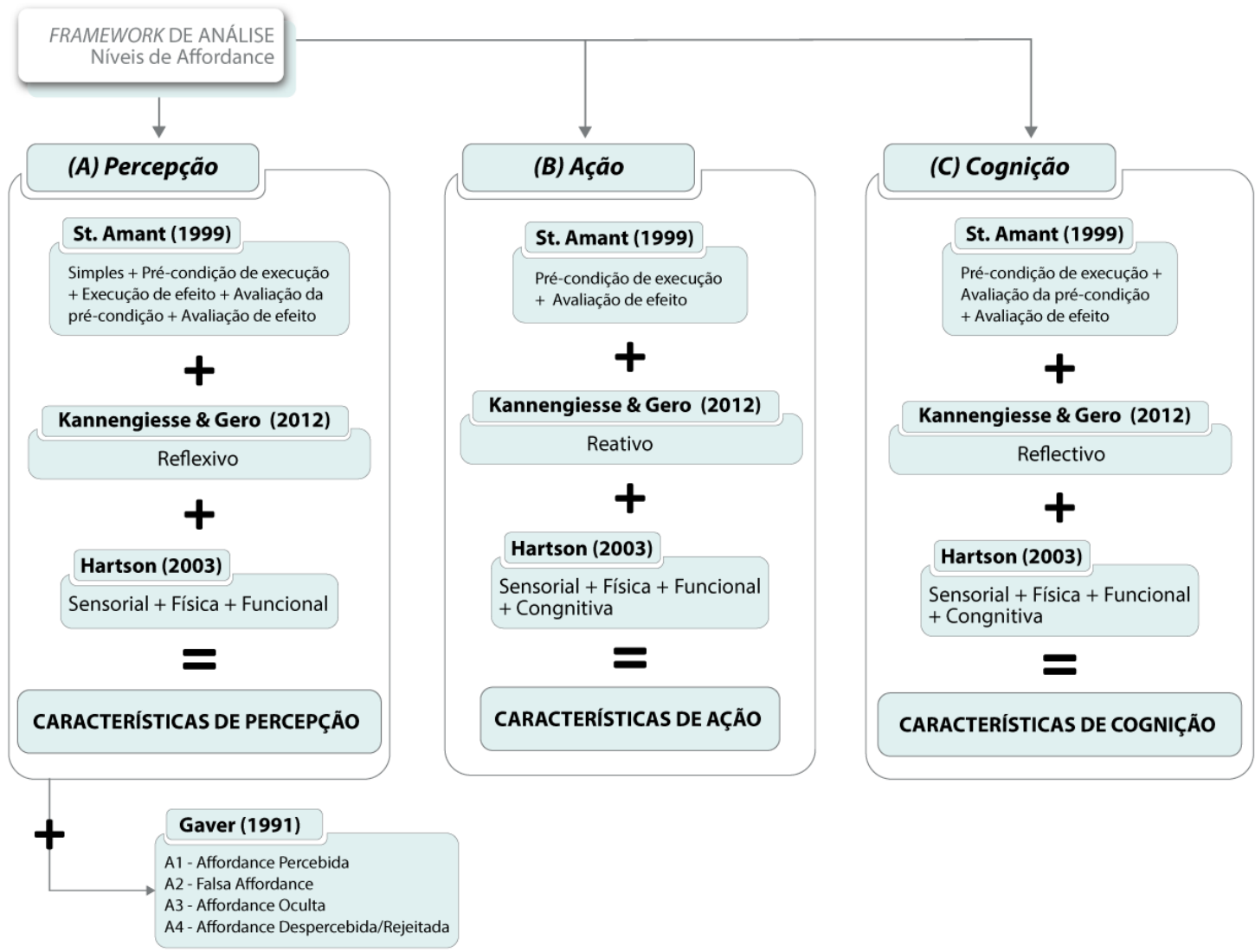

Os níveis de affordance, conforme a figura 4, foram representados em três segmentos: (A) Percepção - a affordance foi percebida e identificada pelos estímulos fornecidos aos usuários; (B) Ação - os usuários reagem, selecionando, testando e avaliando a ação e atuação sobre a affordance; e (C) Cognição - os usuários interpretam, reconhecem, exploram, refletem as diferentes possibilidades de ações da affordance para tomar uma decisão. Por fins de representação, os níveis foram estruturados de acordo com o processamento da informação, 
ou seja, o usuário percebe, tem o comportamento e reflete sobre ação. Porém, não existe o impedimento da (C) Cognição fazer parte do resultado qualquer um dos segmentos.

(2) Etapa prática: Através de um questionário online (via Google Forms) foram coletados relatos sobre as experiências de interação com usuários de interfaces bancárias físicas e digitais. As perguntas do questionário foram estruturadas visando identificar as experiências de interação segmentadas em quatro tópicos: (1) componentes visuais, (2) acionar ou realizar um comando, (3) concluir uma ação ou tarefa, e (4) sentir-se perdido(a) ou encontrar o que precisava. O procedimento de análise ocorreu por intermédio da utilização de um framework teórico para identificar os níveis de affordances: (A) percepção, (B) Ação e (C) Cognição.

(3) Triangulação dos dados: A partir das dificuldades relatadas pelos respondentes do questionário e através da exemplificação da interface foi possível compilar e analisar as informações com base no framework, resultando em reflexões sobre a aplicação de affordances em interfaces bancárias digitais.

\section{Resultados e discussão}

O questionário online foi disponibilizado via redes sociais (Facebook e Linkedin) e via aplicativo de mensagens (WhatsApp) para a rede de contato dos pesquisadores no período entre 24 e 31 de março de 2021. A amostragem foi classificada como não probabilista intencional, e considerou usuários com alguma experiência na utilização de serviços bancários.

Participaram da pesquisa 77 respondentes, sendo 32 concentrados na faixa etária entre 25 a 34 anos; 26 entre 35 a 44 anos; 53 do sexo feminino e 24 do sexo masculino e 56 dos respondentes tinham nível superior completo. A seguir, na Figura 5 é possível visualizar uma síntese dos resultados do estudo.

Figura 5: Síntese dos resultados do questionário | Fonte: Os autores

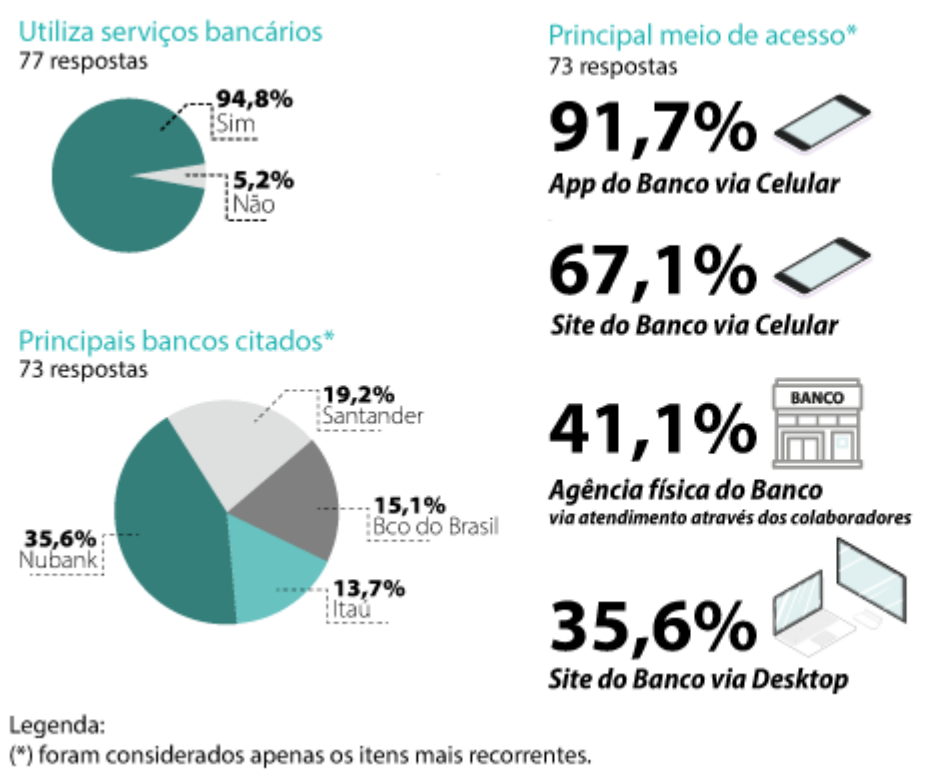

Dificuldades durante a interaçăo com a interface bancária 73 respostas

$42,4 \%$

(1) Entender os componentes visuais

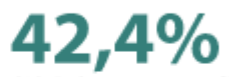

(2) Acionar ou realizar um comando

$45,2 \%$

(3) Concluir uma ação ou tarefa

\section{$39,7 \%$}

(4) Sentir-se perdido(a) ou encontrar o que precisava 
A análise ocorreu a partir da tabulação dos relatos por similaridade dos quatro tópicos do questionário, seguida de uma exemplificação de interface para identificar os níveis de affordances. Com base nos resultados dos principais bancos citados pelos respondentes e pela disponibilidade de acesso à interface, foi definido para a exemplificação o banco Nubank (app mobile).

Com os dados coletados dos respondentes do questionário online, foi possível identificar, em uma visão geral, que a maioria dos usuários não relatou dificuldades em relação às características de percepção, ação, cognição correlacionadas com componentes visuais $(57,6 \%)$, acionar ou realizar um comando $(57,6 \%)$, concluir uma ação ou tarefa $(54,8 \%)$ e não se sentir perdido(a) ou encontrar o que precisava $(60,3 \%)$. À vista disso, foi possível inferir que a interação, de modo geral, resultou em respostas satisfatórias dos estímulos fornecidos pela interface. Por outro lado, existem usuários que relataram dificuldades relacionadas aos 4 tópicos mapeados, sendo possível analisá-los a partir do framework de análise.

Em nenhum momento os respondentes manifestaram desistência da interação. No entanto, existem aspectos da interface que podem impactar essa experiência, seja no nível perceptivo ou cognitivo, tais como: estrutura de menu, botões, caixas de texto, caixas de diálogo, ícones (Galitz, 2007) e na execução dos comandos, como por exemplo, permitir o clique, o movimento de rolagem, entre outros (Rogers, Sharp \& Preece, 2019).

Diante destes níveis o usuário pode não identificar ou entender algum componente de interação, ou agir de forma exploratória para encontrar o comando correto destinado a completar a tarefa. O esforço cognitivo em concluir a tarefa impacta nas ações não planejadas na jornada do usuário, e podem ser resultado da não identificação e compreensão das affordances (Galitz, 2007; Norman, 2013; Rogers, Sharp \& Preece, 2019).

Com base em Hartson (2003), Kannengiesser e Gero (2012) e St. Amant (1999), foi possível classificar os relatos de experiência de interação vivenciados pelos respondentes de acordo com os 4 tópicos do questionário, sintetizados no Quadro 2. 
Quadro 2: Principais aspectos identificados nos relatos | Fonte: Os autores

\begin{tabular}{|c|c|c|}
\hline Aspectos da interface & Dificuldades mapeadas & $\begin{array}{l}\text { Volume de respostas } \\
\text { Total: } 73 \text { respondentes }\end{array}$ \\
\hline $\begin{array}{l}\text { (1) componentes } \\
\text { visuais }\end{array}$ & $\begin{array}{l}\text { compreensão dos componentes gráficos, bem } \\
\text { como a familiaridade do usuário perante a } \\
\text { identificação desses componentes influenciam as } \\
\text { tomadas de decisão, atuação e ações possíveis } \\
\text { de serem realizadas na interface (ex.: aparência } \\
\text { de áreas clicáveis e padronização de ícones) }\end{array}$ & $\begin{array}{l}31 \text { respondentes } \\
(42,4 \%)\end{array}$ \\
\hline $\begin{array}{l}\text { (2) acionar ou realizar } \\
\text { um comando }\end{array}$ & $\begin{array}{l}\text { no acionamento do comando, por exemplo, clicar, } \\
\text { pressionar, arrastar (ações do usuário), e avançar, } \\
\text { retornar, fechar, abrir (resposta da interface) que } \\
\text { estão intrinsecamente relacionadas com o input e } \\
\text { output do processamento de informação (ex.: } \\
\text { retronavegação, falta de feedback informativo) }\end{array}$ & $\begin{array}{l}31 \text { respondentes } \\
(42,4 \%)\end{array}$ \\
\hline $\begin{array}{l}\text { (3) concluir uma ação } \\
\text { ou tarefa }\end{array}$ & $\begin{array}{l}\text { compreensão e falta de informação para realizar } \\
\text { as ações (ex.: hierarquia informacional, arquitetura } \\
\text { da informação, conteúdo informacional) }\end{array}$ & $\begin{array}{l}33 \text { respondentes } \\
(45,2 \%)\end{array}$ \\
\hline $\begin{array}{l}\text { (4) sentir-se } \\
\text { perdido(a) ou } \\
\text { encontrar o que } \\
\text { precisava }\end{array}$ & $\begin{array}{l}\text { compreensão/identificação do agrupamento de } \\
\text { informações dos menus (ex.: } \\
\text { terminologia/legibilidade, menus } \\
\text { minimizados/simplificados) }\end{array}$ & $\begin{array}{l}29 \text { respondentes } \\
(39,7 \%)\end{array}$ \\
\hline
\end{tabular}

Por mais que as perguntas do questionário estivessem segmentadas por tópicos, os respondentes manifestaram suas experiências de forma global, ou seja, em um relato pode ser encontrado referências condizentes com todos os tópicos, conforme as exemplificações a seguir. 
Figura 6: Relatos + exemplificação R70 | Fonte: Os autores, com base em interface mobile do Nubank (2021)

\section{I - Relato do respondente}

"No próprio app do Nubank, não consegui arrastar a barrinha para ver o histórico de compras e me deixou confuso"

Relato do respondente 70

\section{II - Exemplificação da interface}

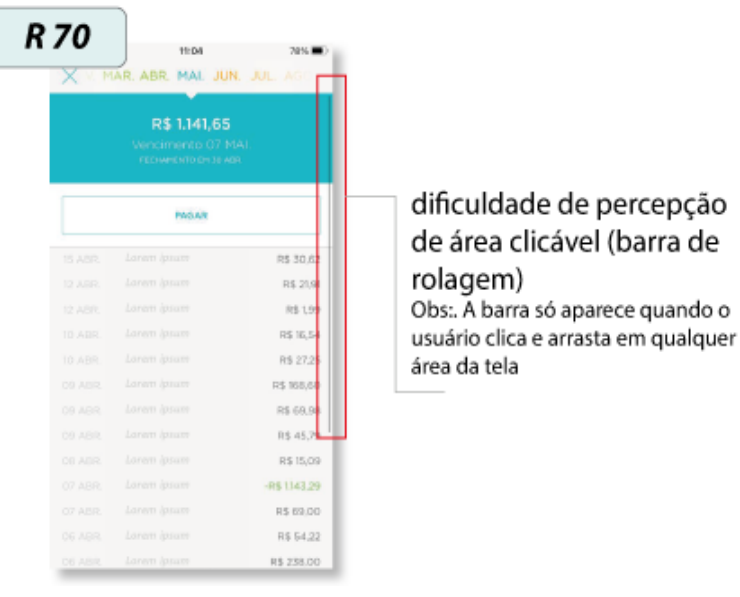

Segundo o relato do R70 houve a percepção e reação perante a informação (barra de rolagem), mas ao acionar um comando (arrastar) não conseguiu concluir corretamente a tarefa. Nesse sentido, a affordance pode ser percebida, mas não condiz com o que o usuário esperava (função da forma representacional - barra), pois em qualquer lugar da tela o usuário consegue arrastar e visualizar a informação, independente do componente barra de rolagem. À vista disso, é possível classificá-la como falsa affordance (Gaver, 1991).

A falsa affordance que apresenta um componente visual perceptivo (barra) e não necessariamente depende dele para executar a ação, faz com que o usuário tente caminhos alternativos do proposto pelo desenvolvedor para entender o que precisa ser feito, resultando em um esforço cognitivo adicional, identificada como pré-condição de execução, conforme St. Amant (1999).

Por mais que a barra de rolagem tenha sido percebida e identificada (reflexivo Kannengiesser \& Gero, 2012) houve dificuldade de compreensão da affordance, localizado no nível Reflectivo de Kannengiesser e Gero (2012). Corroborando com o nível Cognitivo, Sensorial e Funcional de Hartson (2003). Do mesmo modo, a familiaridade com a tecnologia ou a falta de um feedback visual (instrução ou ajuda) também podem comprometer a experiência de interação. 
Figura 7: Relatos + exemplificação R21 e R33 | Fonte: Os autores, com base em interface mobile do Nubank (2021)

\section{I - Relatos dos respondentes}

"na última atualização do app do Nubank, para a opção cartão de crédito aparece inicialmente o valor da fatura e se você quiser saber mais informações como limite, fatura atual ou próxima é preciso rolar para o lado. Mas não tem uma instrução sobre isso, abaixo só uma barrinha mais alongada e ao lado uma bolinha, até eu entender que isso queria dizer que tinha mais coisa do lado...senta que lá vem história!"

Relato do respondente 21

"Dificuldade em encontrar o detalhamento da fatura no app do NuBank"

Relato do respondente 33

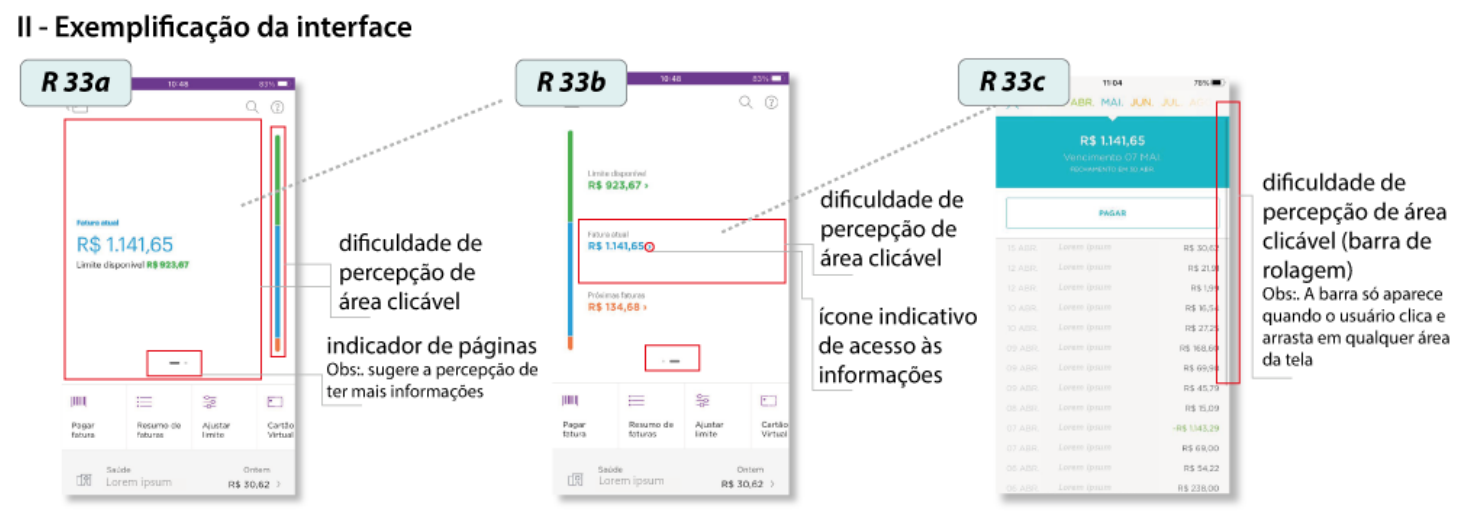

De acordo com as situações relatadas (R33 e R21 - Figura 7) tanto os componentes de navegação quanto a falta de informação comprometem a execução das ações desejadas pelos usuários. A falta de estímulos visuais para que a affordance seja percebida implica no andamento da interação, e novamente requer um esforço cognitivo adicional para reconhecer a pré-condição de execução da affordance (St. Amant, 1999), podendo ser classificada como affordance despercebida/rejeitada (Gaver, 1991).

Ao exemplificar a interface o componente visual pode representar o indicador de páginas (R33a) e pode sugerir ao usuário uma ação para visualizar mais informações, porém requer conhecimento prévio do usuário. Dessa maneira, pode ser classificada no nível reflexivo (Kannengiesser \& Gero, 2012), pois o usuário percebe o estímulo da interface, mas tem dificuldade em compreender a possibilidade de ação, impactando em sua tomada de decisão.

Das possibilidades de ação da affordance, o recurso gráfico que pode ser percebido como um ícone indicativo de acesso à mais informações pode ser classificado em um nível (A) percepção. Este componente visual induz a possibilidade do usuário entender que corresponde a uma área clicável, porém o acionamento pode ocorrer em toda a área branca da tela, incluindo a barra colorida lateral. De certa forma, se o usuário não perceber a informação a affordance também não acontecerá, ou seja, os usuários não conseguirão identificar a área 
clicável e encontrar o detalhamento da fatura (Gaver, 1991; Hartson, 2003; Kannengiesser \& Gero, 2012; St. Amant,1999).

Figura 8: Relatos + exemplificação R51 | Fonte: Os autores, com base em interface mobile do Nubank (2021)

\section{I - Relato do respondente}

"Alguns bancos tem menus muito complicados, confuso por exemplo saber onde eu encontro minhas transações agendadas"

Relato do respondente 51

II - Exemplificação da interface

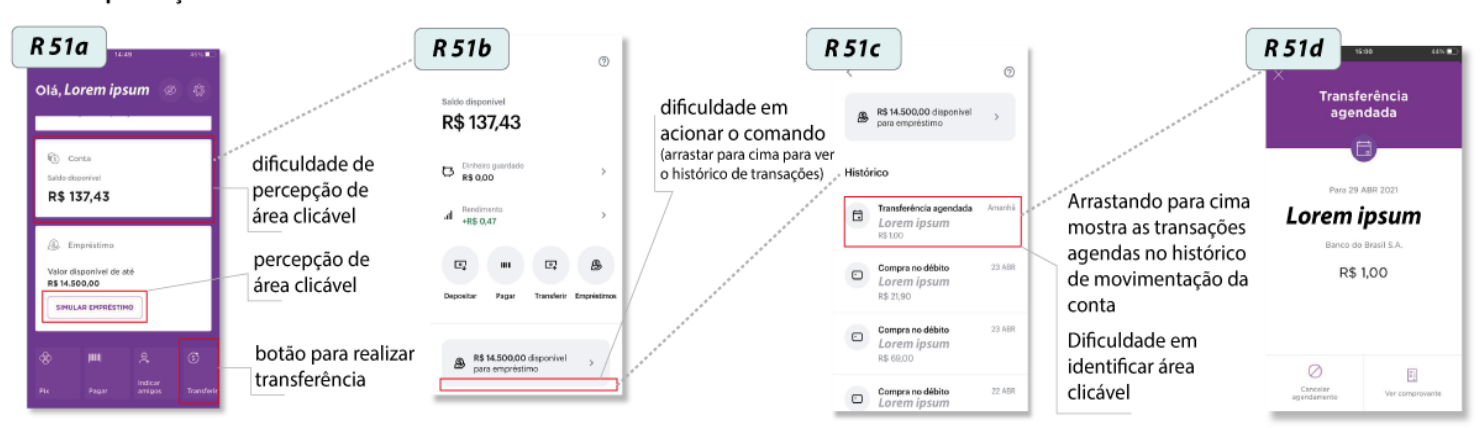

No relato R51 (Figura 8) tanto a informação quanto a affordance podem ser percebidas (Gaver, 1991), mas por questões de hierarquia e organização do conteúdo na interface (ex.: estrutura de menus) o usuário pode se sentir perdido, confuso e suas ações com a interface podem resultar em uma experiência insatisfatória. Logo, podem ser relacionadas com o nível (A) de Percepção e (C) de Cognição, como também, no reconhecimento da pré-condição de execução da affordance (Gaver, 1991; Hartson, 2003; Kannengiesser \& Gero, 2012;

St. Amant, 1999).

Durante a exemplificação da interface foram encontrados aspectos semelhantes às exemplificações anteriores que condizem com a falta de estímulos sensoriais (ex.: ajuda, instrução) e de informações que podem facilitar o reconhecimento da affordance, por exemplo, dificuldade de percepção de área clicável, de execução de ações e comandos, tais exemplos podem ser categorizados no nível reflexivo de Kannengiesser e Gero (2012) e sensorial e funcional de Hartson (2003) e comprometem a avaliação da pré-condição (St. Amant, 1999). 
Figura 9: Relatos + exemplificação R23 | Fonte: Os autores, com base em interface mobile do Nubank (2021)

\section{I - Relato do respondente}

"como mudaram a interface eu não consegui visualizar facilmente o botão de compartilhar o documento após uma transferência. Só após rolar a barra até embaixo e retornar ao topo da página que encontrei. Além disso, o botão era de cor clara e quase imperceptível na tela"

Relato do respondente 23

\section{II - Exemplificação da interface}

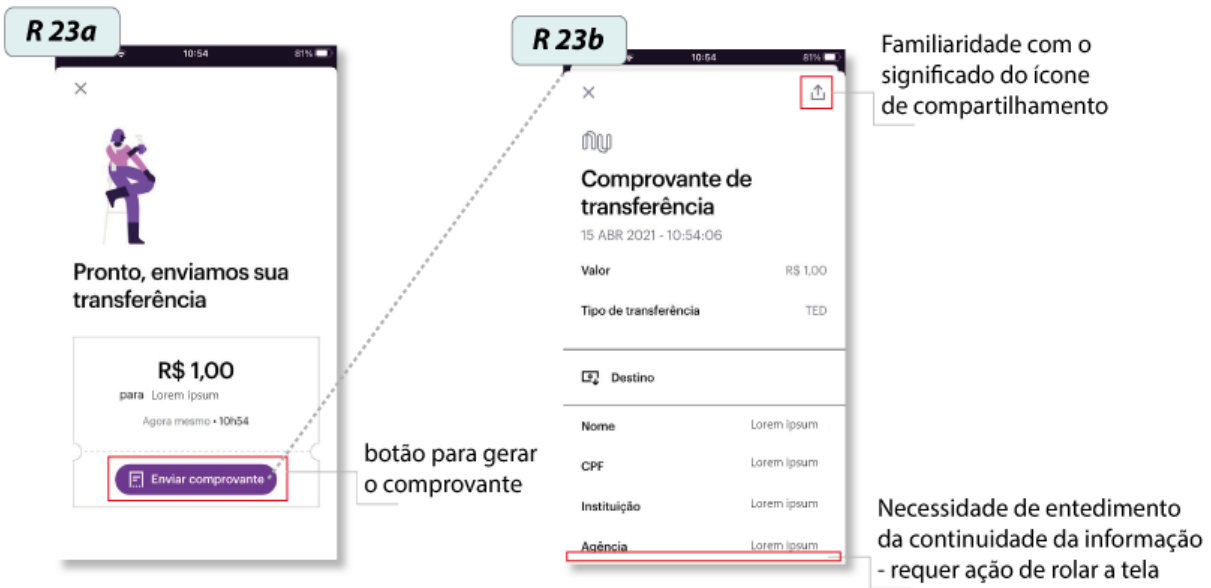

No relato R23 (Figura 9) a atualização da interface pode modificar a diagramação e a hierarquia de informações na tela, com isso, a memorabilidade será comprometida impactando na percepção da informação e o acionamento da affordance, sendo assim, classifica-se no nível (B) de Ação (Gaver, 1991; Hartson, 2003; Kannengiesser \& Gero, 2012; St. Amant,1999).

Durante a exemplificação da interface foi possível verificar que a avaliação da pré-condição (St. Amant, 1999) pode ser mal interpretada pelo usuário, pois a representação visual do ícone de compartilhamento não apresenta recurso visual que o caracteriza como um componente clicável, logo, o botão pode ser identificado como uma barreira para o reconhecimento da affordance.

Outro aspecto verificado na exemplificação da interface condiz com a terminologia e o caminho a ser percorrido para realizar a tarefa de compartilhamento do comprovante. Na figura 10 no item R23a, o botão denomina-se "enviar comprovante", ao executar a ação o usuário obtém como resposta da interface uma nova tela com a geração do comprovante, o que pode ocasionar a confusão de entendimento da ação de compartilhar, já que é necessária a percepção e compreensão do ícone de compartilhar posicionado no canto superior direito da interface para então finalizar a ação.

Dessa forma, os achados podem ser classificados como Reativo (Kannengiesser \& Gero, 2012) e Sensorial, Cognitivo e Funcional (Hartson, 2003). De acordo com o relato R23, apesar da dificuldade encontrada durante a interação devido à falta de familiaridade com a nova interface, houve a percepção da informação e da affordance (affordance percebida - Gaver, 1991). 


\section{Considerações Finais}

O presente artigo teve como objetivo apresentar reflexões acerca da identificação e classificação dos níveis de affordance nas interfaces bancárias. Através do framework de análise foi possível diagnosticar que os níveis de affordance influenciam na percepção e compreensão dos recursos e componentes apresentados na interface.

De modo geral, as affordances são percebidas, porém demandam um nível de esforço cognitivo adicional para que sejam compreendidas e com isso impactam na tomada de decisão para realizar uma ação na interface. Durante a exemplificação da interface alguns aspectos dificultaram o reconhecimento da affordance, por exemplo, a falta de entendimento das informações, componentes da navegação, identificação de área clicável, a falta de estímulos visuais para orientar as ações do usuário.

A partir desse estudo foi possível concluir que as questões de familiaridade com a tecnologia, organização, posicionamento e hierarquia informacional, bem como, a consideração do conhecimento prévio do usuário no desenvolvimento das interfaces pode afetar a percepção e compreensão da informação e da affordance.

Com base na análise dos relatos e na exemplificação da interface foi possível inferir que as escolhas feitas pelo designer influenciarão na percepção dos usuários, impactando no reconhecimento das affordances. Uma possível solução para o designer é considerar a abordagem centrada no usuário, para valorizar aspectos específicos do público-alvo e a aproximação do modelo conceitual com o modelo mental dos usuários.

Portanto, ao considerar o conceito de affordances no desenvolvimento de projetos esperase que os usuários identifiquem as possibilidades de ações de forma intuitiva, a fim de minimizar o esforço cognitivo durante a interação.

Recomenda-se para estudos futuros a aplicação do framework de análise em outros tipos de interfaces, bem como um ensaio de interação presencial para um aprofundamento práticointerativo perante as reflexões dos níveis de affordance.

\section{Agradecimento}

O presente trabalho foi realizado com apoio da Coordenação de Aperfeiçoamento de Pessoal de Nível Superior - Brasil (CAPES) - Código de Financiamento 001.

\section{Referências}

ABNT, A. B. de N. T. (2011). NBR ISO 9241-210 - Ergonomia da interação humano-sistema Parte 210: Projeto centrado no ser humano para sistemas interativos.

Banco Central (2018). Relatório de Cidadania Financeira. Banco Central do Brasil, Brasília - DF.

Dix, A., Finlay, J., Abowd, G. D., \& Beale, R. (2004). Human-computer interaction. Pearson Education Limited. 
Galitz, W. O. (2007). The Essential Guide to User Interface Design: An Introduction to GUI Design Principles and Techniques. Wiley Publishing, Inc.

Gaver, W. W. (1991). Technology Affordances. ACM CHI 91 Human Factors in Computing Systems Conference, 79-84.

Gibson, J. J. (1986). The ecological approach to visual perception (Vol. 1). New York: Psychology Press.

Hartson, R. (2003). Cognitive, physical, sensory, and functional affordances in interaction design. Behaviour \& Information Technology, 22(5), 315-338.

Kannengiesser, U., \& Gero, J. S. (2012). A Process Framework of Affordances in Design. Design Issues, 28(1), 50-62.

McGrenere, J., \& Ho, W. (2000). Affordances: Clarifying and Evolving a Concept. Graphics Interface, May, 1-8.

Norman, D. (2004). Emotional Design: Why we love (or hate) everyday Things. Basic Books.

Norman, D. (2013). The design of everyday things. Revised and expanded edition. Basic Books.

Nubank. (2021). Interface mobile. Disponível em: https://apps.apple.com/br/app/nubank-contae-cart\%C3\%A3o/id814456780. Acesso em: 15 abr 2021.

Rogers, Y., Sharp, H., \& Preece, J. (2019). Interaction Design: beyond human-computer interaction, Fifth Edition, John Wiley \& Sons, Inc.

Shedroff, N. (1999). Information Interaction Design: A Unified Field Theory of Design. In: Jacobson R. (ed) Information Design, MIT Press.

St. Amant, R. (1999). User Interface Affordances in a Planning Representation. HumanComputer Interaction, 14(3), 317-354.

\section{Sobre os autores}

Dominique Leite Adam, Me., UFPR, Brasil <domiadam@gmail.com> André Schlemmer, Me., UFPR, Brasil <schlemmer.andre@gmail.com> Stephania Padovani, Dra., UFPR, Brasil <stephania.padovani@gmail.com> Maria Lucia Leite Ribeiro Okimoto, Pós-doutora, UFPR, Brasil <lucia.demec@ufpr.br> 